\title{
Resveratrol sensitizes A549 cells to irradiation damage via suppression of store-operated calcium entry with Orai1 and STIM1 downregulation
}

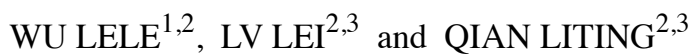 \\ ${ }^{1}$ Department of General Medicine, First People's Hospital of Yuhang, Hangzhou, Zhejiang 311100; \\ ${ }^{2}$ Department of Radiotherapy and ${ }^{3}$ Epigenetic Laboratory, Anhui Provincial Hospital, Hefei, Anhui 230031, P.R. China
}

Received January 4, 2020; Accepted November 26, 2020

DOI: $10.3892 /$ etm.2021.10019

\begin{abstract}
Resveratrol is a natural polyphenol with multiple positive biofunctions and was found to have potential as a radiosensitizer with an intricate molecular mechanism. Store-operated calcium entry (SOCE) is a novel intracellular calcium regulatory pattern that is mainly mediated by iron channels, such as by the stromal interaction molecule (STIM) and calcium release-activated calcium channel protein (Orai) families. SOCE was recently reported to be suppressed via the downregulation of STIM or Orai families for the promotion of tumor cell death induced by resveratrol. In the present study, resveratrol combined with irradiation treatment were found to induce more evident cell damage compared with irradiation treatment alone, as shown with Cell Counting Kit- 8 assay and mitochondrial membrane potential detection with rhodamine 123. Additionally, resveratrol combined with irradiation treatment decreased the expression of STIM1 and Orai1, while it had no effects on STIM2, Orai2 and Orai3. Moreover, resveratrol combined with irradiation treatment lead to alleviated thapsigargin-induced SOCE. In addition, overexpression of STIM1 and Orail reversed resveratrol-induced SOCE inhibition and reduced death in A549 cells under irradiation. In summary, the present results revealed that resveratrol can significantly enhance the effect of irradiation damage on lung adenocarcinoma A549 cells, and this effect may be mediated by suppression of SOCE with reduced expression of both STIM1 and Orail.
\end{abstract}

Correspondence to: Professor Qian Liting, Department of Radiotherapy, Anhui Provincial Hospital, 107 Huanhu East Road, Hefei, Anhui 230031, P.R. China

E-mail: emailqlt@163.com

Key words: radiotherapy, resveratrol, store-operated calcium entry, stromal interaction molecule, calcium release-activated calcium channel protein 1, lung adenocarcinoma, A549 cells

\section{Introduction}

Lung cancer remains the dominant cause of cancer-associated deaths worldwide, and its incidence has been increasing year by year (1). Lung cancer is separated into two clinical types: Small cell lung cancer and non-small cell lung cancer (NSCLC). NSCLC is the most common subtype, accounting for $85 \%$ of confirmed cases (1). For patients with intermediate and advanced lung cancer, who have lost the opportunity for surgery, radiotherapy is one of the most effective treatment methods, which can prolong the survival time of patients (2). However, NSCLC, especially lung adenocarcinoma, is less sensitive to radiotherapy than other types of lung cancer (3). Radiation resistance can significantly affect treatment efficiency. Therefore, research on radiotherapy sensitizers has gradually become a focus in the field of radiobiology study in recent years (4). In this regard, considering the requirements of biosafety, low toxicity, antioxidant properties and public acceptance, natural compounds are regarded as ideal research targets for choosing a sensitizer (5). Resveratrol is a non-flavonoid polyphenol compound extracted from Polygonum cuspidatum, grapes and peanuts (6). It has antitumor, antioxidant, antiaging and immune regulation effects (7). A number of studies have shown that resveratrol has the potential as a radiotherapy sensitizer as it can increase the radiosensitivity of tumor cells and promote tumor cell death via different subcellular pathways (8-10). However, the mechanisms are complex and researchers have not reached a consensus. Recently, studies have found that resveratrol can trigger calcium homeostasis imbalance inside and outside of the cell, thereby reducing mitochondrial membrane potential $(\Delta \psi \mathrm{m})$ and triggering pro-apoptotic protein release, eventually promoting tumor cell death (11-13).

Calcium, as a signal transduction molecule, not only regulates a series of cell activities including transcription, proliferation, migration and death processes but also affects the development and metastasis of tumors (14). There have been an increasing number of studies focusing on store-operated calcium channel (SOCC) and its function in regulating calcium homeostasis (15-17). Store-operated calcium entry (SOCE) mediated by SOCC exists in several types of tumor cells and plays an important role in tumor migration and 
invasion (18). SOCE is an essential way to mediate extracellular $\mathrm{Ca}^{2+}$ to flow inside the cell, following endoplasmic reticulum (ER) calcium depletion regulated by matrix stromal interaction molecule (STIM1) located on the ER, and a calcium release-activated calcium channel protein (Orai1) located on the cell membrane (19). After the depletion of ER calcium storage, STIM1 protein can sense the change in $\mathrm{Ca}^{2+}$ concentration through its structure and activate reactions such as rapid translocation and polymerization (20). STIM1 subsequently couples with Orai1 protein on the plasma membrane to achieve functional opening of the SOCE pathway and promote $\mathrm{Ca}^{2+}$ influx from the outside (21). When $\mathrm{Ca}^{2+}$ is replenished in the calcium pool, STIM1 protein and Orail protein are slowly dissociated and inactivated, and the pathway is closed. In mammals, STIM proteins often express two homologs, STIM1 and STIM2, and Orai has three homologs including Orai1, Orai2 and Orai3 (22). Resveratrol activated autophagic cell death in prostate, colorectal and myeloma cancer cells via the downregulation of STIM1 and SOCE $(23,24)$. However, to the best of our knowledge, the effects of resveratrol on SOCE or SOCC in NSCLC have not been thoroughly studied.

The present study aimed at investigating the effects of resveratrol on the radiosensitization of lung adenocarcinoma A549 cells and demonstrating whether it can regulate SOCE activity to achieve the sensitization effect by affecting STIM or Orai expression. The findings may provide a basis for improving radiotherapy effects and prognosis of patients with advanced lung cancer.

\section{Materials and methods}

Cell culture, drug administration and radiation therapy. Lung adenocarcinoma A549 cells were kindly donated by The Cell Bank of Type Culture Collection of The Chinese Academy of Sciences. Cells were cultured in DMEM (Gibco; Thermo Fisher Scientific, Inc.) containing a mixture of $10 \%$ fetal bovine serum (Gibco; Thermo Fisher Scientific, Inc.), $1 \%$ penicillin and $1 \%$ streptomycin (Gibco; Thermo Fisher Scientific, Inc.) at $37^{\circ} \mathrm{C}$ and $5 \% \mathrm{CO}_{2}$, as previously described (9). A549 cells at the logarithmic growth phase were seeded (6x $10^{5}$ cells/well) into six-well culture dishes. Once the cells have adhered, resveratrol (Sigma-Aldrich; Merck KGaA) was dissolved in dimethylsulfoxide (Sigma-Aldrich; Merck KGaA) to produce a stock solution of $10,000 \mu \mathrm{M}$, and stored at $-20^{\circ} \mathrm{C}$. Different concentrations of resveratrol $(0,10,50,100$, 150 and $200 \mu \mathrm{mol} / \mathrm{l})$ were added into each dish and incubated for $24 \mathrm{~h}$ at $37^{\circ} \mathrm{C}$, and the resveratrol-containing medium was removed with a pipette. The same resveratrol treatment groups were prepared and irradiated with $0,2,4$ and 6 Gy X-rays (room temperature, $6 \mathrm{MV}$ X-rays, $300 \mathrm{cGy} / \mathrm{min}$, the distance from the source to the specimen was $100 \mathrm{~cm}$ and the size of the irradiation field was $20 \times 20 \mathrm{~cm})$. The culture was continued for $4 \mathrm{~h}$ at $37^{\circ} \mathrm{C}$ after irradiation for the following experiments.

Cell counting. Cell counting was performed to perform an approximate evaluation of cell damage from irradiation and resveratrol treatment. In total, $0.8 \times 10^{6}$ A549 cells were seeded into 60-mm culture dish to conduct the following experiment. After receiving corresponding experimental treatments, the culture medium was replaced to wash out dead cells and the remaining A549 cells growing on the bottom of the $60-\mathrm{mm}$ culture dish were placed under a light microscope (magnification, $\mathrm{x} 40$ ) to check the overall growth state. In order to quantify the cell number, A549 cells were resuspended in 1X PBS and $100 \mu \mathrm{l}$ cell suspension was added to the corresponding chip, which was inserted into the cell counting machine (Scepter2.0; Sigma-Aldrich; Merck KGaA) in order to calculate the concentration of the remaining cells within each 100- $\mu$ 1 A549 cell suspension. Together, the data were used to calculate the total remaining A549 cell number within an entire 50-mm culture dish.

Cell counting Kit-8 (CCK-8) assay. CCK-8 assay was performed to check the cell viability after respective experimental treatments. A549 cells in the logarithmic growth phase were dissociated with trypsin and $5 \times 10^{3}$ cells/well were added to a 96-well plate. Once the cells had adhered to the well, they were divided into different groups and treated with various concentrations of resveratrol and different doses of irradiation, as aforementioned. After incubation at $5 \% \mathrm{CO}_{2}$ and $37^{\circ} \mathrm{C}$ for $4 \mathrm{~h}, 10 \mu \mathrm{l} \mathrm{CCK}-8$ solution (Beyotime Institute of Biotechnology) was added to each well. After $2 \mathrm{~h}$ of incubation, the optical density of each well was measured at a wavelength of $450 \mathrm{~nm}$ on an enzyme-linked immunosorbent detector (Beyotime Institute of Biotechnology).

$\Delta \psi m$. A549 cells $\left(2.5 \times 10^{5}\right.$ cells $\left./ \mathrm{ml}\right)$ were seeded onto each well of a 24-well plate and incubated for $24 \mathrm{~h}$. After treatment with different doses of resveratrol $(0,10,50,100,150$ and 200 $\mu \mathrm{M})$ combined with different doses of irradiation $(0,2,4$ and $6 \mathrm{~Gy})$, the culture medium was removed from each well and the cells were washed twice with PBS at room temperature. Rhodamine 123 powder (Dalian Meilun Biology Technology Co., Ltd.) was prepared into a $1 \mathrm{mg} / \mathrm{ml}$ stock solution with methanol and stored at $-20^{\circ} \mathrm{C}$. The cells were incubated with $5 \mu \mathrm{g} / \mathrm{ml}$ rhodamine 123 for $30 \mathrm{~min}$ in a $5 \% \mathrm{CO}_{2}$ cell incubator at room temperature, then washed with PBS two or three times, and finally $0.5 \mathrm{ml}$ PBS was added to each well. The plate was placed under a fluorescent microscope (Nikon M50; Nikon Corporation) with parameters initially set to detect fluorescence of rhodamine 123 (480/40 excitation filter, 505 long pass dichroic mirror and 535/40 emission filter; magnification, $\mathrm{x} 20$ ) and red fluorescence dots indicated mitochondria inside the cells (10). Briefly, once a clear visual field with 200-300 cells is achieved, images were captured at five different and qualified visual fields randomly within one well for each experimental group. The fluorescence intensity was reversely associated with $\Delta \psi \mathrm{m}$ and mean fluorescent intensity within an image was analyzed using Image J 1.8.0 software (National Institutes of Health).

Western blotting. RIPA buffer (Beyotime Institute of Biotechnology) was used to extract total cell protein in different dose groups, and the protein concentration was determined spectrophotometrically using a BCA assay (Beyotime Institute of Biotechnology). After boiling and denaturing, $30 \mu \mathrm{g}$ proteins were separated by $10 \%$ SDS-PAGE and transferred to a PVDF film (EMD Millipore). TBS-T (50 mM Tris- $\mathrm{HCl}$, $\mathrm{pH} 7.6,150 \mathrm{mM} \mathrm{NaCl}$, and $0.1 \%$ Tween-20) containing $5 \%$ skim milk was used to block the membranes for $2 \mathrm{~h}$ room 
temperature. The following primary antibodies (diluted 1:1,000; all ProteinTech Group, Inc.), were added and incubated at $4^{\circ} \mathrm{C}$ overnight: Rabbit anti-human STIM1 (cat. no. 11565-1-AP), STIM2 (cat. no. 21192-1-AP), mouse anti-human Orail (cat. no. 66223-1-lg), Orai2 (cat. no. 20592-1-AP) and Orai3 (cat. no. 25766-1-AP). $\beta$-actin (cat. no. 66009-1-Ig) were used as an internal control. The following day, TBST was used to wash the membrane for $10 \mathrm{~min}$, and HRP-labeled anti-rabbit or anti-mouse antibodies were added (1:2,000; cat. nos. SA00001-1 and 11565-1-AP, respectively; ProteinTech Group, Inc.). After $1 \mathrm{~h}$ incubation at room temperature, the membranes were washed for 10 min three times with PBS-T, and the bands were visualized using an ECL development gel imaging system (ImageQuant LAS 500; Cytiva). Each experiment was repeated at least three times. Densitometry for each graph was conducted using ImageJ 1.8.0 software (National Institutes of Health).

Calcium imaging. After experimental treatment, a round coverslip was placed on the bottom of a 12-well plate using tweezers, and $150 \mu \mathrm{l} 100 \mu \mathrm{g} / \mathrm{ml}$ polylysine was added to the middle of each coverslip. The polylysine was then removed by pipette after $10 \mathrm{~min}$. A total of $4 \times 10^{4}$ cells were seeded on circular coverslips. Following incubation for $8 \mathrm{~h}$ with culture medium, $10 \mu \mathrm{mol} / 1$ Fluo-8 AM (cat. no. ab112129; Abcam), a fluorescent probe for intracellular $\mathrm{Ca}^{2+}\left(\left[\mathrm{Ca}^{2+}\right] \mathrm{i}\right)$, was added and incubated at $37^{\circ} \mathrm{C}$ for $30 \mathrm{~min}$. The sample was washed twice with $\mathrm{Ca}^{2+}$-free PBS buffer (standard external solution including $10 \mu \mathrm{M}$ HEPES, $120 \mu \mathrm{M} \mathrm{NaCl}, 5.4 \mu \mathrm{M} \mathrm{KCl}, 1 \mu \mathrm{M}$ $\mathrm{MgCl}_{2}$ and $10 \mu \mathrm{M}$ glucose; $\mathrm{pH}$ 7.4) and calcium fluorescence was detected under a fluorescent microscope with MetaFluor software 7.0 (Molecular Devices, LLC) (magnification, x200). Briefly, stained cells were initially observed under white light, by adjusting the focus, opening the fluorescence and detecting the cells in darkness. The software tools were used to capture each cell outline within the visual field and their fluorescence intensity was recorded. When the fluorescence intensity curve become stable for $3 \mathrm{~min}, \mathrm{Ca}^{2+}$ release was induced by $2 \mu \mathrm{mol} / 1$ thapsigargin (cat. no 12758; Cell Signaling Technologies, Inc.) administration and left for another $5 \mathrm{~min}$ until the fluorescent curve become stable. Extracellular $\mathrm{Ca}^{2+}$ solution (cat. no. c1016; Sigma-Aldrich; Merck KGaA; 1 mmol/l) was then added to induce calcium influx with anther fluorescent peak and left for 5 min until the fluorescent curve became stable again. The process was observed under a fluorescence microscope (Nikon M50; Nikon Corporation) at $488 \mathrm{~nm}$ excitation and $515 \mathrm{~nm}$ emission wavelengths (magnification, $\mathrm{x} 200)$. The change in $\left[\mathrm{Ca}^{2+}\right] \mathrm{i}$ was compared with the fluorescence intensity (F0) before adding extracellular $\mathrm{Ca}^{2+}$. SOCE was demonstrated as the ratio of peak intensity $(\mathrm{F} 1) / \mathrm{F} 0$ and the intensity of each fluorescence data was an average of 20-30 cells (11). Data were collected and analyzed with MetaFluor software7.0 (Molecular Devices LLC).

Cell transfection. The Orail cDNA sequence was inserted into a pCMVPuro01 expression vector (Guangzhou Sinogen Pharmaceutical Co., Ltd.). The forward and reverse primer sequences for constructing the vectors were 5'-CTAGTC TAGAATGCATCCGGAGCCCGCCC-3' and 5'-TCCTTC GAACTAGGCATAGTGGCTGCCGG-3'. The STIM1 cDNA sequence was also inserted into a pCMVPuro01 expression vector (Guangzhou Sinogen Pharmaceutical Co., Ltd.). The forward and reverse primer sequences for constructing the vectors were 5'-AAGAGTCTACCGAAGCAG-3' and 5'-GTG CTATGTTTCACTGTTGG-3'. Empty vectors were used as a negative control. Briefly, an appropriate number of cells $\left(4 \times 10^{4}\right)$ were seeded into each well (12-well dish) with $1 \mathrm{ml}$ medium and incubated at $37^{\circ} \mathrm{C}$ and $5 \% \mathrm{CO}_{2}$ until the cells reached $80-90 \%$ confluence for transfection. Orail overexpression vector (100 pmol) and/or STIM1 overexpression vector $(100 \mathrm{pmol})$ were added to $100 \mu \mathrm{l}$ Opti-MEM I reducing serum medium (Invitrogen; Thermo Fisher Scientific, Inc.) within an Eppendorf tube, and $5 \mu$ l Lipofectamine ${ }^{\circledR} 3000$ (Invitrogen; Thermo Fisher Scientific, Inc.) was added to the same tube. The tube was gently agitated and incubated at room temperature for $25 \mathrm{~min}$ to form Lipofectamine complexes. The old medium was aspirated, and $1 \mathrm{ml}$ serum-free medium was added. The Lipofectamine complex $(100 \mu \mathrm{l})$ was added to each well and mixed gently. The cells were incubated at $37^{\circ} \mathrm{C}$ and $5 \% \mathrm{CO}_{2}$ for $8 \mathrm{~h}$ and the serum-free medium was replaced with normal medium. Transfection efficiency was assessed via western blotting after $48 \mathrm{~h}$.

Statistical analysis. All experiments were repeated independently at least three times. Data are presented as the mean \pm SD. Multiple comparison analysis was conducted using one-way ANOVA followed by Tukey's post hoc test. $\mathrm{P}<0.05$ was considered to indicate a statistically significant difference. All analyses were performed with GraphPad Prism 5.0 (GraphPad Software, Inc.) and SPSS 19.0 (IBM Corp.).

\section{Results}

Effects of resveratrol combined with irradiation treatment on proliferation and apoptosis of A549 cells. A549 cells at the logarithmic growth phase were divided into different experimental groups, receiving a series of concentrations of resveratrol $(0,10,50,100,150$ and $200 \mu \mathrm{mol} / \mathrm{l})$ combined with different doses $(0,2,4$ and $6 \mathrm{~Gy})$ of X-ray irradiation. After experimental treatment, cell growth was observed, and cell numbers were counted and compared under a light microscope. The data showed that increased cell death and reduced cell numbers depended on resveratrol and irradiation dose. For each irradiation dose, compared with the $0 \mu \mathrm{M}$ Res group, a significant cell number reduction was observed initially from $100 \mu \mathrm{M}$ resveratrol treatment group. Among all the irradiation doses with $100 \mu \mathrm{M}$ resveratrol, a significant cell number reduction was observed between 2 and $4 \mathrm{~Gy}$. Although combination of higher doses of irradiation and resveratrol also significantly reduced the cell number, based on the principle that the least but significant dosage might be the best, the combination of $4 \mathrm{~Gy}$ irradiation and $100 \mu \mathrm{M}$ resveratrol could be used as an ideal treatment strategy for the following experiments (Fig. 1A). CCK-8 assay was further used to detect A549 cell viability in different dose groups. Similar to light microscopy results, irradiation promoted the reduction of cell viability, and resveratrol pretreatment enhanced this effect (Fig. 1B). The $\Delta \psi \mathrm{m}$ is a key parameter of mitochondrial function, and its decrease is often associated with apoptosis (25). Therefore, the fluorescent lipophilic cationic dye rhodamine 123 was used for $\Delta \psi \mathrm{m}$ detection 
in each group. As a positively charged dye, rhodamine 123 will accumulate in the mitochondria in an inverse proportion to the $\Delta \psi \mathrm{m}$. The accumulation of fluorescent dyes in mitochondria was detected by confocal microscopy to evaluate $\Delta \psi \mathrm{m}$ to compare apoptosis (26). Fluorescent microscopy showed that both resveratrol and irradiation treatment reduced the $\Delta \psi \mathrm{m}$ of A549 cells in a dose-dependent manner and the combination of resveratrol and irradiation enhanced this effect (Fig. 2A and B), which was also consistent with aforementioned experiments (Fig. 1).

Effects of resveratrol combined with irradiation treatment on thapsigargin-induced SOCE in lung adenocarcinoma A549 cells. The aforementioned results indicated that resveratrol had the potential to be developed as an irradiation sensitizer. Thus, in order to further investigate the subcellular mechanism, the effects of resveratrol combined with irradiation treatment on thapsigargin-induced SOCE in lung adenocarcinoma A549 cells were detected using calcium imaging in four experimental groups: Blank control, 4 Gy irradiation, $100 \mu \mathrm{M}$ resveratrol and 4 Gy irradiation $+100 \mu \mathrm{M}$ resveratrol using Fluo-8, an intracellular calcium fluorescent probe. When the fluorescence intensity curve was detected to be stable, thapsigargin was utilized to cause calcium storage depletion, and the level of intracellular calcium rapidly increased and then decreased to normal levels. Subsequently, extracellular calcium was added to trigger SOCE, which demonstrated another instant increase in intracellular fluorescence (Fig. 3B). Based on the results, the peak level of fluorescence intensity was significantly reduced by resveratrol and this reduction was enhanced when combined with irradiation, indicating that SOCE was significantly inhibited by the addition of resveratrol (Fig. 3A and C).

Effects of resveratrol combined with irradiation treatment on SOCCs in lung adenocarcinoma A549 cells. STIM and Orai family members are the SOCCs mediating the SOCE process in non-excited cells, responsible for inflow, storage and consumption of calcium ions (13). Thus, western blotting was used to detect the expression of STIM1, STIM2, Orai1, Orai2 and Orai3 proteins in four experimental groups: Blank control, 4 Gy irradiation, $100 \mu \mathrm{M}$ resveratrol and 4 Gy irradiation $+100 \mu \mathrm{M}$ resveratrol. The results showed that compared with controls, resveratrol alone or resveratrol combined with irradiation treatment had no significant effect on the expression of STIM2, Orai2 and Orai3 in A549 cells but significantly suppressed the expression of STIM1 and Orail. Among the four groups, combined treatment exerted the strongest effects (Fig. 4A and B). To some extent, these results indicated that resveratrol-sensitized irradiation damage in A549 cells may be associated with STIM1 and Orai1 downregulation that leads to SOCE suppression.

Overexpression of STIMI and Orail partly reduces resveratrol-sensitized lung adenocarcinoma A549 cell damage under irradiation. Downregulation of SOCE was associated with apoptosis in various cell types, including osteosarcoma, skin cancer and renal cancer (27-29). According to the aforementioned results, it was found that resveratrol can inhibit SOCE by downregulating the expression of STIM1 and
A

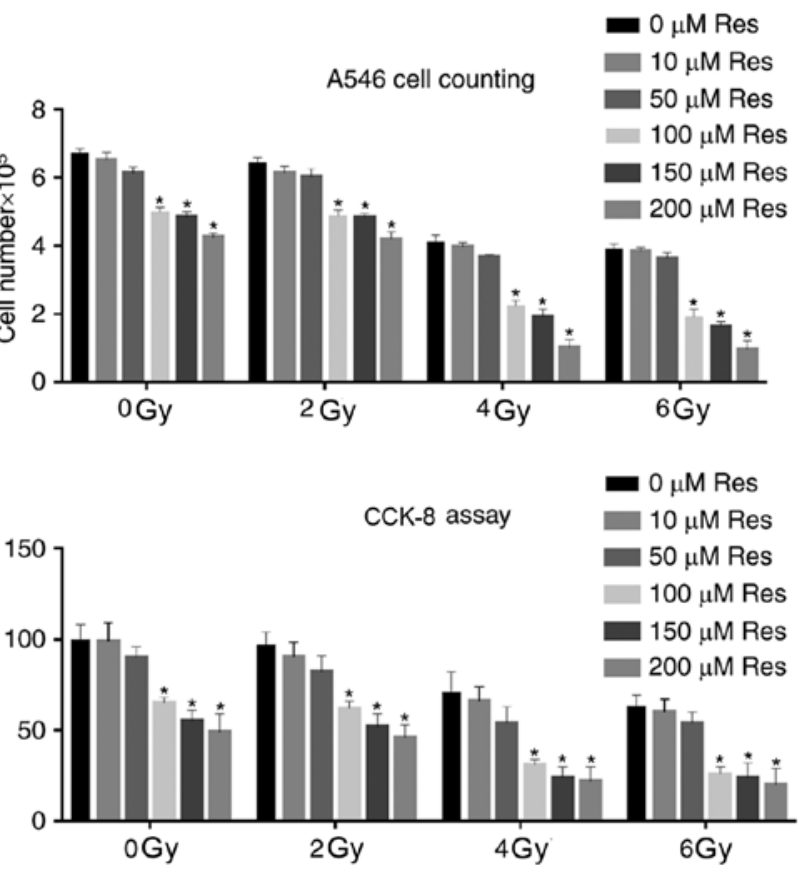

Figure 1. Effects of a range of doses of resveratrol and irradiation treatment on the overall survival state of A549 cells. Different concentrations of resveratrol $(0,10,50,100,150$ and $200 \mu \mathrm{M})$ combined with different doses of irradiation $(0,2,4$ and 6 Gy) were used to treat lung cancer A549 cells. (A) General survival states of cells in each experimental group were observed and numbers of cells under different treatments were detected using a cell counter. (B) Cell viability in each group was detected via CCK-8 assay. The results are presented as the ratio of absorbance relative to that of the untreated group. Data are presented as the mean \pm standard deviation. $n=5$. ${ }^{*} \mathrm{P}<0.05$ vs. $0 \mu \mathrm{M}$ Res at each respective dose of radiation. Res, resveratrol; CCK-8, Cell Counting Kit-8.

Orai1, further leading to A549 cell death. To further verify this result, STIM1 and Orai1 were overexpressed, following resveratrol treatment and irradiation, in order to observe whether the recovery of STIM1 and Orail could restore SOCE and cell death in A549 cells. Western blotting images (Fig. S1A and B) and corresponding analysis (Fig. S1C and D) demonstrated that the vectors were transfected successfully. The expression levels of STIM1 and Orai1 proteins, following respective or combined treatment with Orail and STIM1 overexpression vectors after administration of both resveratrol and irradiation, were significantly increased compared with the pure combined treatment group with resveratrol and irradiation (Fig. 5A and B). Calcium imaging (Fig. 6A and B) and corresponding analysis of SOCE (Fig. 6C) in each group were partly consistent with our hypothesis. Although the results were not statistically significant, the SOCE of the combined resveratrol and irradiation treatment group was partly restored after adding both STIM1 and Orail overexpression vectors compared with single treatment groups. Additionally, CCK-8 results indicated that cell viability of the resveratrol combined with irradiation treatment group was partly restored after adding both STIM1 and Orai1 overexpression vectors compared with single treatment groups (Fig. 5C). Based on the aforementioned findings, the role of resveratrol in sensitizing A549 cells to irradiation treatment may be associated with downregulating the expression of STIM1 and Orail, inhibiting the SOCE process and triggering cell death. 


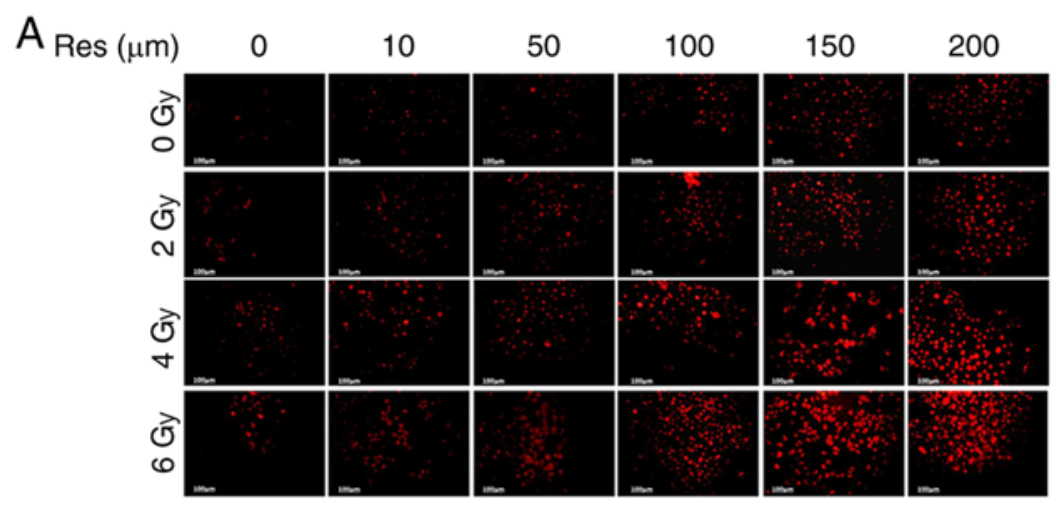

B

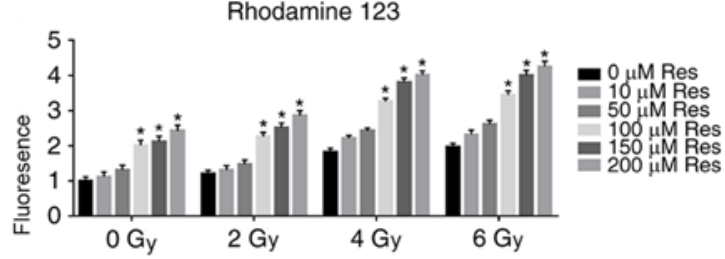

Figure 2. Effects of a range of doses of resveratrol and irradiation treatment on the mitochondrial membrane potential of A549 cells. Different concentrations of resveratrol $(0,10,50,100,150$ and $200 \mu \mathrm{M})$ combined with different doses of irradiation $(0,2,4$ and $6 \mathrm{~Gy})$ were used to treat lung cancer A549 cells. (A) Fluorescence staining images of rhodamine 123 and (B) corresponding mitochondrial membrane potential analysis presenting the changes in the mitochondrial membrane potential after each experimental treatment. The higher the fluorescence intensity, the higher the degree of mitochondrial membrane potential depolarization. Magnification, $\mathrm{x} 200$. The results are presented as the ratio of absorbance relative to that of the untreated group. Data are presented as the mean \pm standard deviation. $\mathrm{n}=5$. ${ }^{*} \mathrm{P}<0.05$ vs. $0 \mu \mathrm{M}$ Res at each respective dose of radiation. Res, resveratrol.

A

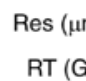

RT (Gy)
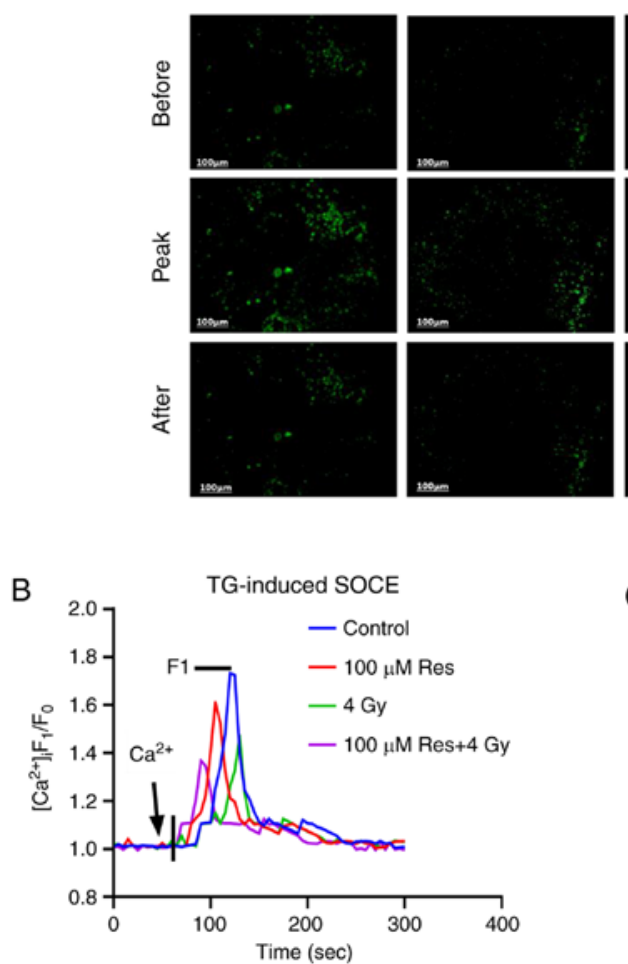

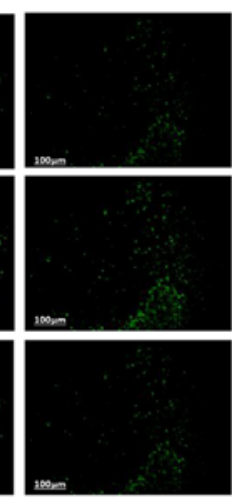

100

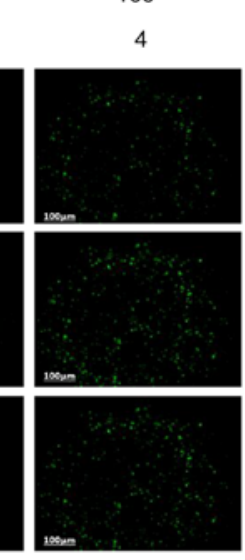

C

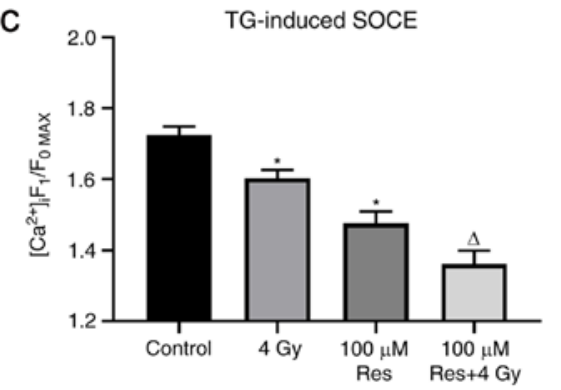

Figure 3. Effects of a range of doses of resveratrol and irradiation treatment on SOCE in A549 cells. Lung cancer A549 cells were treated with $100 \mu \mathrm{M}$ resveratrol, $4 \mathrm{~Gy}$ irradiation or both, and untreated. $\left[\mathrm{Ca}^{2+}\right]$ i was labeled with sensitive fluorescent indicator Fluo- $8 \mathrm{AM}$ and its real-time fluorescence intensity was detected. After $\mathrm{Ca}^{2+}$ storage depletion in A549 cells of different dose groups induced by $2 \mu \mathrm{mol} / 1 \mathrm{TG}, 1 \mathrm{mmol} / 1$ extracellular Ca ${ }^{2+}$ was added to trigger SOCE. (A) Representative fluorescence images, (B) fluorescence intensity curve and (C) summarized data of SOCE are shown. The change in [Ca $\left.{ }^{2+}\right] \mathrm{i}$ was illustrated as the ratio of $\mathrm{F} 1$ and $\mathrm{F} 0$. SOCE was demonstrated as the $\mathrm{F} 1 / \mathrm{FO}_{\max }$ and the intensity of fluorescence was an average of 20-30 cells. Magnification, $\mathrm{x} 200$. Values are shown as the mean \pm standard deviation. $n=3$. ${ }^{*} \mathrm{P}<0.05$. vs. control; ${ }^{\wedge} \mathrm{P}<0.05$. vs. $4 \mathrm{~Gy}$. Before, images of Fluo-8-labeled A549 cells before addition of extracellular $\mathrm{Ca}^{2+}$; Peak, images of Fluo-8-labeled A549 cells giving off strongest fluorescence following addition of extracellular Ca ${ }^{2+} ;$ After, images of Fluo-8-labeled A549 cells at 5 min after addition of extracellular $\mathrm{Ca}^{2+}$; STOCE, store-operated calcium entry; RT, radiotherapy; TG, thapsigargin; Res, resveratrol; [Ca $\left.{ }^{2+}\right]$ i, intracellular $\mathrm{Ca}^{2+} ; \mathrm{F} 1$, real-time fluorescence intensity; $\mathrm{F} 0$, fluorescence intensity before the addition of extracellular calcium; $\mathrm{F} 1 / \mathrm{F} 0_{\max }$, ratio of peak intensity. 

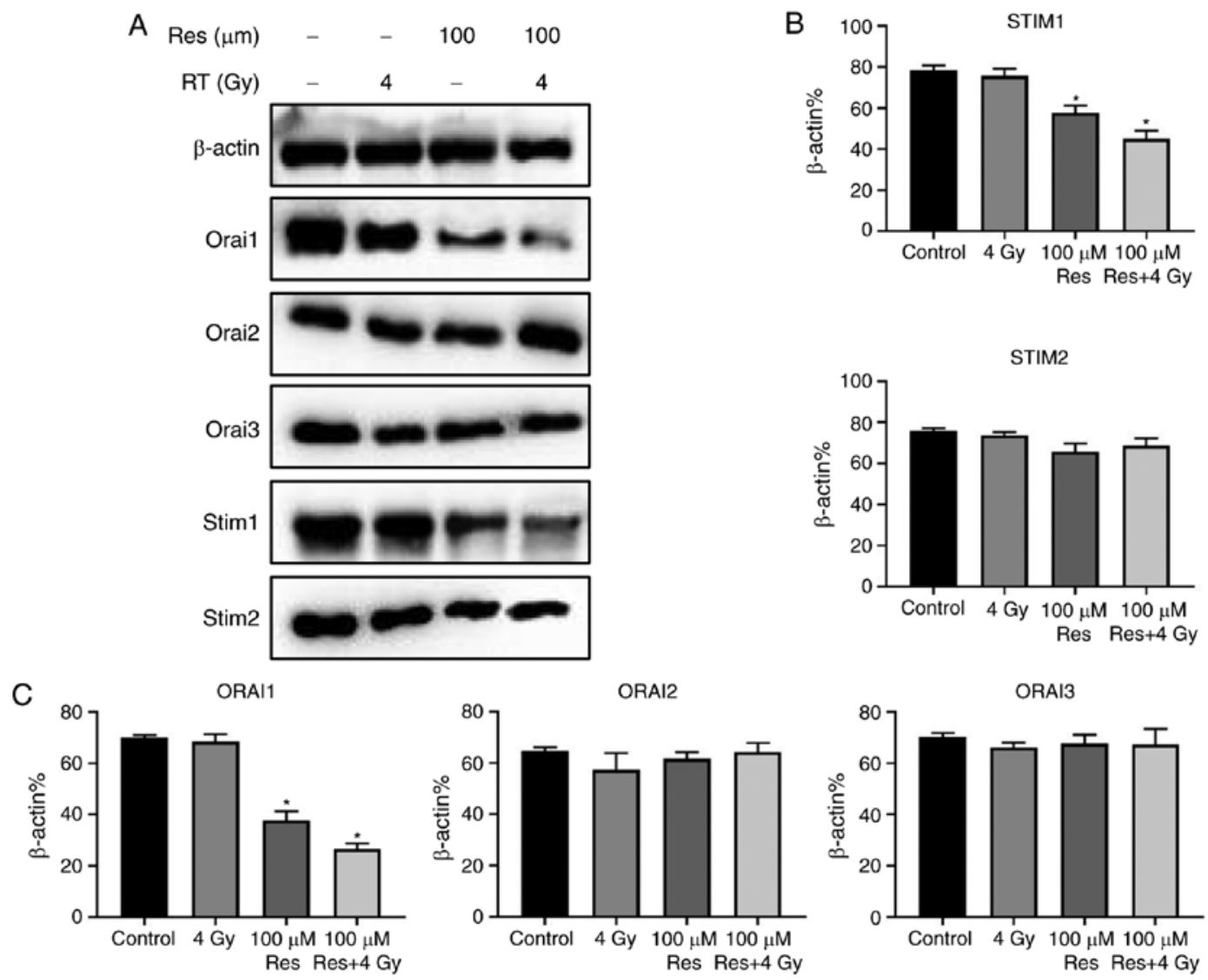

Figure 4. Combined effects of resveratrol and irradiation treatment on Orai and STIM family members in A549 cells. (A) Different concentrations of resveratrol $(0$ and $100 \mu \mathrm{M})$ combined with different doses of irradiation ( 0 and $4 \mathrm{~Gy})$ were used to treat lung cancer A549 cells. Subsequently, total proteins were extracted, and western blotting was performed. Representative images and analysis showing the expression levels of (B) STIM1, STIM2, (C) Orai1, Orai2 and Orai3. $\beta$-actin was used as a loading control. Values are shown as the mean \pm standard deviation. ${ }^{*} \mathrm{P}<0.05$. $\mathrm{n}=3$. STIM, stromal interaction molecule; Orai, calcium release-activated calcium channel protein; Res, resveratrol.

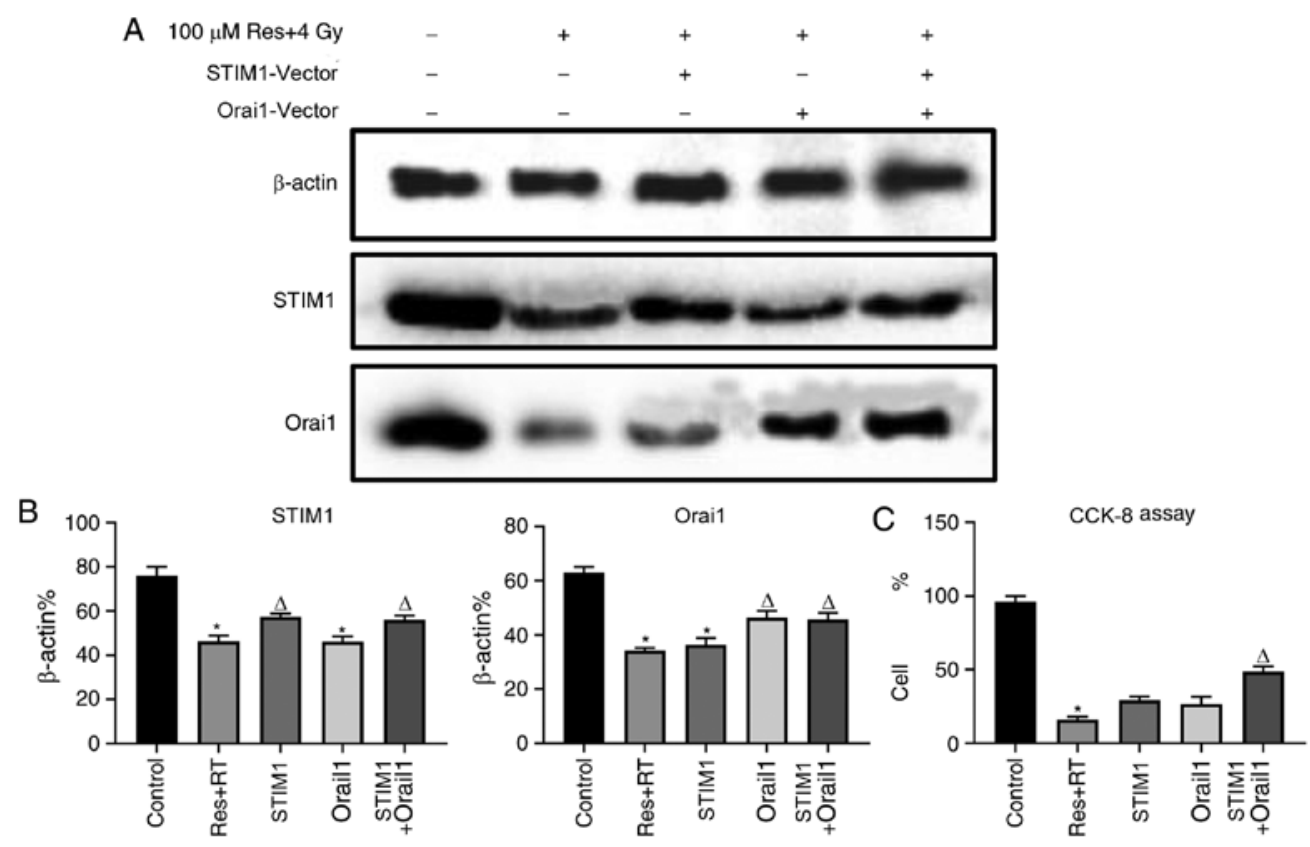

Figure 5. Effects of various doses of resveratrol and irradiation treatment following Orai1, STIM1 or dual overexpression of STIM1 or Orai1 in A549 cells. After the cells were cultured to the logarithmic phase, they were divided into five experimental groups: Untreated, 4 Gy irradiation $+100 \mu \mathrm{M}$ resveratrol, $4 \mathrm{~Gy}$ irradiation $+100 \mu \mathrm{M}$ resveratrol + STIM1 overexpression vector, 4 Gy irradiation + $100 \mu \mathrm{M}$ resveratrol + Orail overexpression vector group and 4 Gy irradiation $+100 \mu \mathrm{M}$ resveratrol + STIM1 + Orail overexpression vector. Following treatment, western blotting was used to detect the levels of STIM1 and ORAI1 protein expression in each group. (A) Corresponding images and (B) analysis show the expression levels of STIM1 and Orail protein after experimental treatment. (C) Cell viability in each group was detected using a CCK- 8 assay. Values are shown as the mean \pm standard deviation $n=3$. ${ }^{*} \mathrm{P}<0.05$. vs. control. ${ }^{\Delta} \mathrm{P}<0.05$. vs. Res $+\mathrm{RT}$. RT, radiotherapy; STIM, stromal interaction molecule; CCK-8, Cell Counting Kit-8; Orai, calcium release-activated calcium channel protein; Res, resveratrol. 

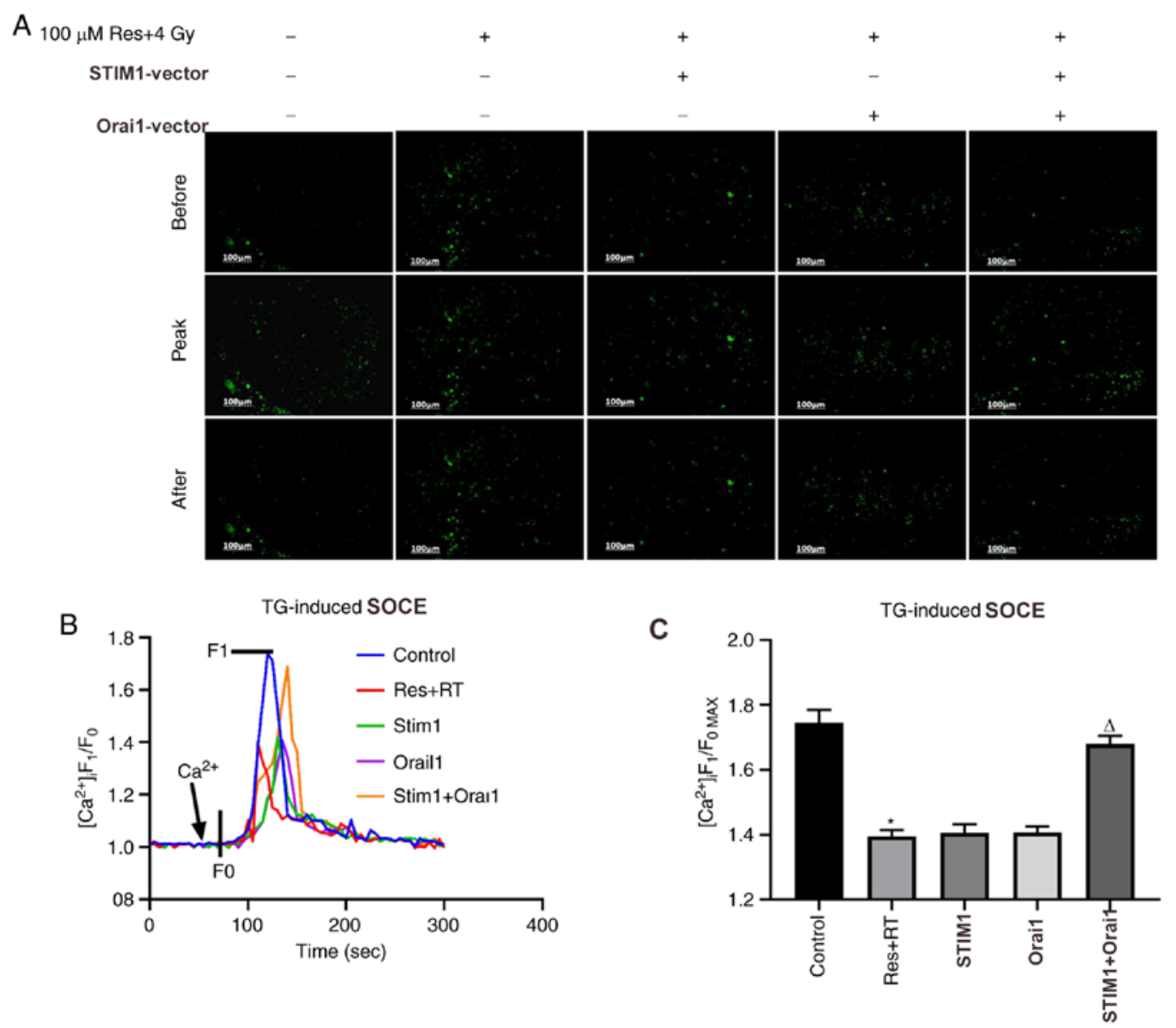

Figure 6. Effects of various doses of resveratrol and irradiation treatment following Orai1, STIM1 or dual overexpression on SOCE in A549 cells. After the cells were cultured to the logarithmic phase, they were divided into five experimental groups: Untreated, 4 Gy irradiation $+100 \mu \mathrm{M}$ resveratrol, 4 Gy irradiation + $100 \mu \mathrm{M}$ resveratrol + STIM1 overexpression vector, $4 \mathrm{~Gy}$ irradiation $+100 \mu \mathrm{M}$ resveratrol + Orail overexpression vector group and $4 \mathrm{~Gy}$ irradiation $+100 \mu \mathrm{M}$ resveratrol + STIM1 + Orail overexpression vector group. $\left[\mathrm{Ca}^{2+}\right]$ i was labeled with sensitive fluorescence indicator Fluo-8 AM and its real-time fluorescent intensity was detected. Following $\mathrm{Ca}^{2+}$ store depletion in lung cancer A549 cells of different dose groups induced by the addition of $2 \mu \mathrm{mol} / 1 \mathrm{TG}$, extracellular calcium was added to observe the SOCE. (A) Representative fluorescence images, (B) fluorescence intensity curves and (C) summarized data of SOCE are shown. The change in $\left[\mathrm{Ca}^{2+}\right] \mathrm{i}$ is illustrated as the ratio of $\mathrm{F} 1$ and $\mathrm{F} 0$. SOCE was demonstrated as the $\mathrm{F} 1 / \mathrm{F} 0_{\max }$ and the intensity of fluorescence data is an average of 20-30 cells. Magnification, $x 200$. Values are shown as the mean \pm standard deviation. $n=3$. ${ }^{*} \mathrm{P}<0.05$. vs. control; ${ }^{\wedge} \mathrm{P}<0.05$. vs. Res $+\mathrm{RT}$. Before, images of Fluo-8-labeled A549 cells before addition of extracellular $\mathrm{Ca}^{2+} ;$ Peak, images of Fluo-8-labeled A549 cells giving off strongest fluorescence following addition of extracellular $\mathrm{Ca}^{2+}$; After, images of Fluo-8-labeled A549 cells at 5 min after addition of extracellular $\mathrm{Ca}^{2+}$; SOCE, store-operated calcium entry; STIM, stromal interaction molecule; Orai, calcium release-activated calcium channel protein; RT, radiotherapy; Res, resveratrol; TG, thapsigargin; [Ca $\left.{ }^{2+}\right]$ i, intracellular $\mathrm{Ca}^{2+} ; \mathrm{F} 1$, real-time fluorescence intensity; $\mathrm{F} 0$, fluorescence intensity before the addition of extracellular calcium; $\mathrm{F} 1 / \mathrm{FO} 0_{\max }$, ratio of peak intensity.

\section{Discussion}

Most patients with NSCLC are diagnosed at advanced stages (30). Traditionally, for inoperable stage III NSCLC and other medically inoperable diseases, high-dose external radiotherapy is generally considered to be a suitable treatment for the disease (31). However, in terms of long-term tumor control, the effects of radiation therapy have been poor (32). The median survival of patients receiving radiation therapy is $<10$ months, and the 5 -year survival rate varies between 5 and $10 \%$. The two main reasons for its low efficacy are limited radiosensitivity of the tumor and the presence of systemic micrometastases at the time of diagnosis (1). Therefore, enhancing the sensitivity of NSCLC is essential.

In order to confirm whether resveratrol had radiosensitization effects on lung adenocarcinoma A549 cells, the present study used microscopic observation, cell counting, CCK- 8 assay and $\Delta \psi \mathrm{m}$ detection to compare cell damage in different dose groups of resveratrol combined with radiation.
Compared with single resveratrol or irradiation treatment, enhanced cell death occurred in the resveratrol combined irradiation treatment group, indicating that resveratrol enhanced the irradiation damage of lung adenocarcinoma A549 cells. Considering that the optimal dose combination should be as little as possible but also trigger maximum cell damage, experiments found that the radiation dose of 4 Gy combined with $100 \mu \mathrm{M}$ resveratrol was the most suitable choice for following experiments. An increase in cell damage was observed following 2 to $4 \mathrm{~Gy}$ irradiation and 50 to $100 \mu \mathrm{M}$ resveratrol administration. Western blotting results showed that the expression levels of STIM1 and Orail in the resveratrol combined with irradiation treatment group were evidently downregulated compared with the single resveratrol or irradiation treatment group, while little effects were observed on STIM2, Orai2 and Orai3. Concurrently, in the calcium imaging experiment, SOCE in the resveratrol combined with irradiation treatment group was significantly inhibited. 
Aside from resveratrol, irradiation may also influence SOCC expression and activity of SOCE, and it has been previously reported that radiation inhibited salivary gland function by promoting STIM1 cleavage by caspase-3 and loss of SOCE via a transient receptor potential cation channel subfamily $M$ member 2-dependent pathway (19). However, to the best of our knowledge, no other study has illustrated the effects of irradiation on SOCE, and the present results indicated that compared with the control group, irradiation itself did not result in statistically significant up- or downregulation on STIM, Orai family members and SOCE activity, indicating that loss of SOCE, STIM1 and Orai1 was exclusively caused by adding resveratrol. Therefore, it was concluded that the effects of resveratrol-sensitized irradiation damage were associated with downregulating the expression of STIM1 and Orai1 and subsequent loss of SOCE process. To further verify this result, an overexpression vector was constructed to reverse the effects. The results of western blotting and calcium imaging experiments showed that the effects of resveratrol combined irradiation treatment were restored to a certain extent after co-overexpression of both STIM1 and Orai1, although it was not completely reversed. This experiment determined that resveratrol could sensitize A549 cells to irradiation damage, and this effect may be associated with the downregulation of STIM1 and Orail expression, leading to the inhibition of SOCE, and then triggering cell death.

Published studies demonstrated that the mechanism of resveratrol-sensitized irradiation damage mainly includes: i) Affecting the balance of pro- and anti-apoptotic molecules, promoting the increase of ROS, affecting tumor cell metabolism and promoting autophagy to induce tumors cell death (33-36); ii) inhibition of related cyclins, transcription factors and pathways to inhibit proliferation (37-39); iii) mediating anti-inflammatory and anti-angiogenic effects to inhibit tumorigenesis and development (40-43); and iv) protection of surrounding tissues to decrease the side effects of radiation (44-46). However, few studies associated this mechanism with $\operatorname{SOCE}(37,47,48)$. The present study proposed a new hypothesis and verified that the effects of resveratrol-sensitized irradiation damage may be associated with SOCE. Compared with single treatment, resveratrol combined with irradiation treatment significantly affected the expression of STIM1 and Orai1, and these two proteins are associated with SOCE. Intracellular calcium serves a number of regulatory functions as an intracellular bio-signal messenger (49). When the ER releases $\mathrm{Ca}^{2+}$, STIM1 can sense $\mathrm{Ca}^{2+}$ consumption, triggering structural changes and combine with Orail to form $\mathrm{Ca}^{2+}$ channels, guide extracellular $\mathrm{Ca}^{2+}$ inflow and compensate for $\mathrm{Ca}^{2+}$ consumption. Compared with the single irradiation treatment group, the resveratrol combined irradiation treatment group significantly downregulated the expression of STIM1 and Orai1, leading to a decrease in calcium channels, decrease in calcium influx and depletion of calcium storage, thereby causing calcium imbalance inside the cell leading to cell dysfunction, and eventually cell death (Figs. 1-3). At present, there are few studies indicating that the target of resveratrol may be associated with ERK1/2. When intracellular calcium is reduced to a certain limit, STIM1 protein is activated (50). This activation is mainly achieved through the phosphorylation of STIM1 protein at Ser575, Ser608 and Ser621, which are ERK1/2 target sites (51). Under the quiescent state, STIM1 binds to end-binding protein 1 , a major regulator of microtubule growing ends. ERK1/2 can promote STIM1 unbinding activation during calcium consumption, polymerize SOCC in plasma membrane and activate SOCE (52). Resveratrol can interfere with SOCE by inhibiting ERK1/2 activity and inhibiting STIM1 phosphorylation (53).

Apart from cell or animal level investigations, numerous resveratrol-based clinical trials have been performed and found to have promising effects on cancer inhibition $(54,55)$. This therapeutic effect was found to exist in a wide range of human cancer types including bowel, breast, bladder, prostate, liver, thyroid and lung, with a $0.5-5$-g daily oral intake of resveratrol (56). It was reported that plasma levels of $14.7 \mu \mathrm{mol} / 1$ resveratrol led to slightly suppressed hepatic cancer metastasis in patients with colorectal cancer, which is substantially lower than the $100 \mu \mathrm{mol} / 1$ dose used in the present study, indicating that more studies may be required to determine the most effective dosage of resveratrol (57).

Although resveratrol was shown to have a radiosensitizing effect, its poor water solubility and low stability limit its application (58). New derivatives are required to be further developed by researchers to enhance their preventive and therapeutic efficiency. The present study only used resveratrol for cell-level research and animal experiments and clinically relevant resveratrol and irradiation treatment cases were not presented. Therefore, the in vivo therapeutic effect need to be further studied.

In conclusion, this study demonstrated that resveratrol can significantly enhance the effect of irradiation damage on lung adenocarcinoma A549 cells, and this effect was related with suppression of SOCE caused by reduction of both STIM1 and Orai1. As a safe bioactive antioxidant, resveratrol has already been applied in health care and cosmetic industry. Once its sensitizing effects, mechanism and pharmacokinetic feature are confirmed in animal level and random clinical trials, this beneficial polyphenol can be quickly put into practical pretreatment ahead of radioactive therapy for cancer patients to minimize the dose-dependent irradiation damage without disturbing the efficacy.

\section{Acknowledgements}

The authors would like to thank Dr Jinsen Lu (Department of Orthopedics of Anhui Provincial Hospital; Hefei, China), who instructed on training group members to detect the fluctuation of intracellular calcium intensity and SOCE via fluorescent microscopy.

\section{Funding}

This study was supported by a grant from the Key Research and Development Projects of Anhui Province (grant no. 1704a0802157).

\section{Availability of data and materials}

The datasets used and/or analyzed during the current study are available from the corresponding author on reasonable request. 


\section{Authors' contributions}

WL, LL and QL designed the experiments. WLL and LL performed the experiments and the data analysis. WLL contributed to data acquisition and analysis. WLL drafted the manuscript. All authors read and approved the final manuscript.

\section{Ethics approval and consent to participate}

Not applicable.

\section{Patient consent for publication}

Not applicable.

\section{Competing interests}

The authors declare that they have no competing interests.

\section{References}

1. Atagi S, Kawahara M, Yokoyama A, Okamoto H, Yamamoto N Ohe Y, Sawa T, Ishikura S, Shibata T, Fukuda H, et al: Thoracic radiotherapy with or without daily low-dose carboplatin in elderly patients with non-small-cell lung cancer: A randomised, controlled, phase 3 trial by the Japan Clinical Oncology Group (JCOG0301). Lancet Oncol 13: 671-678, 2012.

2. Jonna S and Subramaniam DS: Molecular diagnostics and targeted therapies in non-small cell lung cancer (NSCLC) An update. Dis Med 27: 167-170, 2019.

3. Brown S, Banfill K, Aznar MC, Whitehurst P and Faivre Finn C: The evolving role of radiotherapy in non-small cell lung cancer. Br J Radiol 92: 20190524, 2019.

4. Li YL, Hu X, Li QY, Wang F, Zhang B, Ding K, Tan BQ, Lin NM and Zhang C: Shikonin sensitizes wild-type EGFR NSCLC cells to erlotinib and gefitinib therapy. Mol Med Rep 18: 3882-3890, 2018.

5. Crooker K, Aliani R, Ananth M, Arnold L, Anant S and Thomas SM: A review of promising natural chemopreventive agents for head and neck cancer. Cancer Prev Res (Phila) 11: 441-450, 2018.

6. Breuss JM, Atanasov AG and Uhrin P: Resveratrol and its effects on the vascular system. Int J Mol Sci 20: 1523, 2019.

7. Athar M, Back JH, Kopelovich L, Bickers DR and Kim AL: Multiple molecular targets of resveratrol: Anti-carcinogenic mechanisms. Arch Biochem Biophys 486: 95-102, 2009.

8. Ko JH, Sethi G, Um JY, Shanmugam MK, Arfuso F, Kumar AP, Bishayee A and Ahn KS: The role of resveratrol in cancer therapy. Int J Mol Sci 18: 2589, 2017.

9. Jiang Z, Chen K, Cheng L, Yan B, Qian W, Cao J, Li J, Wu E, Ma Q and Yang W: Resveratrol and cancer treatment: Updates. Ann N Y Acad Sci 1403: 59-69, 2017.

10. Vervandier-Fasseur D and Latruffe N: The potential use of resveratrol for cancer prevention. Molecules 24: 4506, 2019.

11. Pan X, Chen J, Wang W, Chen L, Wang L, Ma Q, Zhang J, Chen L, Wang G, Zhang M, et al: Resveratrol-induced antinociception is involved in calcium channels and calcium/caffeine-sensitive pools. Oncotarget 8: 9399-9409, 2017.

12. Xu H, Cheng J, Wang X, Liu H, Wang S, Wu J, Xu B, Chen A and He F: Resveratrol pretreatment alleviates myocardial ischemia/reperfusion injury by inhibiting STIM1-mediated intracellular calcium accumulation. J Physiol Biochem 75: 607-618, 2019.

13. Stephan LS, Almeida ED, Markoski MM, Garavaglia J and Marcadenti A: Red Wine, resveratrol and atrial fibrillation. Nutrients 9: 1190, 2017

14. So CL, Saunus JM, Roberts-Thomson SJ and Monteith GR: Calcium signalling and breast cancer. Semin Cell Dev Biol 94: 74-83, 2019.

15. Wang Y, He J, Jiang H, Zhang Q, Yang H, Xu X, Zhang C, $\mathrm{Xu} \mathrm{C}$, Wang $\mathrm{J}$ and $\mathrm{Lu} \mathrm{W}$ : Nicotine enhances store-operated calcium entry by upregulating HIF-1 $\alpha$ and SOCC components in non-small cell lung cancer cells. Oncol Rep 40: 2097-2104, 2018
16. Tang X, Qian LL, Dang SP, Wu Y, Liu XY, Zhang ZY, Miu LF and Wang RX: Effects of n-3 polyunsaturated fatty acid on store-operated calcium channels in coronary artery smooth muscle cells derived from diabetic rat. Zhonghua Xin Xue Guan Bing Za Zhi 47: 640-646, 2019 (In Chinese).

17. Wu H: Calcium signaling in platelet activation. Sheng Li Ke Xue Jin Zhan 43: 417-421, 2012 (In Chinese).

18. Pan Z and Ma J: Open Sesame: Treasure in store-operated calcium entry pathway for cancer therapy. Sci China Life Sci 58: 48-53, 2015.

19. Ambudkar IS, de Souza LB and Ong HL: TRPC1, Orai1, and STIM1 in SOCE: Friends in tight spaces. Cell Calcium 63: 33-39, 2017.

20. Lopez JJ, Jardin I, Sanchez-Collado J, Salido GM, Smani T and Rosado JA: TRPC Channels in the SOCE Scenario. Cells 9: 126, 2020.

21. Rosenberg P, Katz D and Bryson V: SOCE and STIM1 signaling in the heart: Timing and location matter. Cell Calcium 77: 20-28, 2019.

22. Prakriya M and Lewis RS: Store-operated calcium channels. Physiol Rev 95: 1383-1436, 2015.

23. Collins HE, Zhu-Mauldin X, Marchase RB and Chatham JC: STIM1/Orai1-mediated SOCE: Current perspectives and potential roles in cardiac function and pathology. Am J Physiology Heart Circ Physiol 305: H446-H458, 2013.

24. Lopez E, Frischauf I, Jardin I, Derler I, Muik M, Cantonero C, Salido GM, Smani T, Rosado JA and Redondo PC: STIM1 phosphorylation at $\mathrm{Y}^{316}$ modulates its interaction with SARAF and the activation of SOCE and $I_{\mathrm{CRAC}}$. J Cell Sci 132: jcs226019, 2019.

25. Ren X, Zhang L, Zhang Y, Mao L and Jiang H: Mitochondria response to camptothecin and hydroxycamptothecine-induced apoptosis in Spodoptera exigua cells. Pestic Biochem Physiol 140: 97-104, 2017.

26. Perry SW, Norman JP, Barbieri J, Brown EB and Gelbard HA: Mitochondrial membrane potential probes and the proton gradient: A practical usage guide. Biotechniques 50: 98-115, 2011.

27. Xie J, Pan H, Yao J, Zhou Y and Han W: SOCE and cancer: Recent progress and new perspectives. Int J Cancer 138: 2067-2077, 2016.

28. Jardin I and Rosado JA: STIM and calcium channel complexes in cancer. Biochim Biophys Acta 1863: 1418-1426, 2016.

29. Tanwar J and Motiani RK: Role of SOCE architects STIM and orai proteins in cell death. Cell Calcium 69: 19-27, 2018.

30. Vokes EE, Govindan R, Iscoe N, Hossain AM, San Antonio B, Chouaki N, Koczywas M and Senan S: The impact of staging by positron-emission tomography on overall survival and progression-free survival in patients with locally advanced NSCLC. J Thorac Oncol 13: 1183-1188, 2018.

31. Velcheti V, Chandwani S, Chen X, Pietanza MC and Burke T: First-line pembrolizumab monotherapy for metastatic PD-L1-positive NSCLC: Real-world analysis of time on treatment. Immunotherapy 11: 889-901, 2019.

32. Wu YL, Yang JC, Kim DW, Lu S, Zhou J, Seto T, Yang JJ, Yamamoto N, Ahn MJ, Takahashi T, et al: Phase II study of crizotinib in east asian patients with ROS1-positive advanced non-small-cell lung cancer. J Clin Oncol 36: 1405-1411, 2018.

33. Liu X, Gong B, de Souza LB, Ong HL, Subedi KP, Cheng KT, Swaim W, Zheng C, Mori Y and Ambudkar IS: Radiation inhibits salivary gland function by promoting STIM1 cleavage by caspase-3 and loss of SOCE through a TRPM2-dependent pathway. Sci Signal 10: eaal4064, 2017.

34. Voellger B, Waldt N, Rupa R, Kirches E, Melhem O, Ochel HJ, Mawrin $\mathrm{C}$ and Firsching R: Combined effects of resveratrol and radiation in GH3 and TtT/GF pituitary adenoma cells. J Neurooncol 139: 573-582, 2018.

35. Fang Y, Bradley MJ, Cook KM, Herrick EJ and Nicholl MB: A potential role for resveratrol as a radiation sensitizer for melanoma treatment. J Surg Res 183: 645-653, 2013.

36. Tak JK, Lee JH and Park JW: Resveratrol and piperine enhance radiosensitivity of tumor cells. BMB Rep 45: 242-246, 2012.

37. Chen YA, Lien HM, Kao MC, Lo UG, Lin LC, Lin CJ, Chang SJ, Chen CC, Hsieh JT, Lin H, et al: Sensitization of radioresistant prostate cancer cells by resveratrol isolated from arachis hypogaea stems. PLoS One 12: e0169204, 2017.

38. Tan Y, Wei X, Zhang W, Wang X, Wang K, Du B and Xiao J: Resveratrol enhances the radiosensitivity of nasopharyngeal carcinoma cells by downregulating E2F1. Oncol Rep 37: 1833-1841, 2017.

39. Assad DX, Borges GA, Avelino SR and Guerra ENS: Additive cytotoxic effects of radiation and mTOR inhibitors in a cervical cancer cell line. Pathol Res Pract 214: 259-262, 2018. 
40. Said RS, El-Demerdash E, Nada AS and Kamal MM: Resveratrol inhibits inflammatory signaling implicated in ionizing radiation-induced premature ovarian failure through antagonistic crosstalk between silencing information regulator 1 (SIRT1) and poly(ADP-ribose) polymerase 1 (PARP-1). Biochem Pharmacol 103: 140-150, 2016.

41. Choi YJ, Heo K, Park HS, Yang KM and Jeong MH: The resveratrol analog HS-1793 enhances radiosensitivity of mouse-derived breast cancer cells under hypoxic conditions. Int J Oncol 49: 1479-1488, 2016.

42. Aravindan S, Natarajan M, Herman TS, Awasthi V and Aravindan N: Molecular basis of 'hypoxic' breast cancer cell radio-sensitization: Phytochemicals converge on radiation induced Rel signaling. Radiat Oncol 8: 46, 2013.

43. Firouzi F, Khoei S and Mirzaei HR: Role of resveratrol on the cytotoxic effects and DNA damages of iododeoxyuridine and megavoltage radiation in spheroid culture of U87MG glioblastoma cell line. Gen Physiol Biophys 34: 43-50, 2015.

44. Sener TE, Tavukcu HH, Atasoy BM, Cevik O, Kaya OT, Cetinel S, Dagli Degerli A, Tinay I, Simsek F, Akbal C, et al: Resveratrol treatment may preserve the erectile function after radiotherapy by restoring antioxidant defence mechanisms, SIRT1 and NOS protein expressions. Int J Impot Res 30: 179-188, 2018.

45. Xu L, Yang X, Cai J, Ma J, Cheng H, Zhao K, Yang L, Cao Y, Qin Q, Zhang C, et al: Resveratrol attenuates radiation-induced salivary gland dysfunction in mice. Laryngoscope 123: E23-E29, 2013.

46. Zhang H, Zhai Z, Wang Y, Zhang J, Wu H, Wang Y, Li C, Li D, Lu L, Wang X, et al: Resveratrol ameliorates ionizing irradiation-induced long-term hematopoietic stem cell injury in mice. Free Radic Biol Med 54: 40-50, 2013.

47. Feng Y,Zhou J and Jiang Y: Resveratrol in lung cancer-a systematic review. J BUON 21: 950-953, 2016

48. Yousef M, Vlachogiannis IA and Tsiani E: Effects of resveratrol against lung cancer: In vitro and in vivo studies. Nutrients 9 : 1231, 2017.

49. Boon N, Hul GB, Viguerie N, Sicard A, Langin D and Saris WH: Effects of 3 diets with various calcium contents on 24-h energy expenditure, fat oxidation, and adipose tissue message RNA expression of lipid metabolism-related proteins. Am J Clin Nutr 82: 1244-1252, 2005.
50. Chen X, Hu X, Li Y, Zhu C, Dong X, Zhang R, Ma J, Huang S and Chen L: Resveratrol inhibits Erk1/2-mediated adhesion of cancer cells via activating PP2A-PTEN signaling network. J Cell Physiol 234: 2822-2836, 2019.

51. Thiel G and Rössler OG: Resveratrol stimulates c-Fos gene transcription via activation of ERK1/2 involving multiple genetic elements. Gene 658: 70-75, 2018.

52. Soltoff SP and Lannon WA: Activation of ERK1/2 by store-operated calcium entry in rat parotid acinar cells. PLoS One 8: e72881, 2013.

53. Casas-Rua V, Alvarez IS, Pozo-Guisado E and Martín-Romero FJ Inhibition of STIM1 phosphorylation underlies resveratrol-induced inhibition of store-operated calcium entry. Biochem Pharmacol 86: 1555-1563, 2013.

54. Rauf A, Imran M, Butt MS, Nadeem M, Peters DG and Mubarak MS: Resveratrol as an anti-cancer agent: A review. Crit Rev Food Sci Nutr 58: 1428-1447, 2018.

55. Carter LG, D'Orazio JA and Pearson KJ: Resveratrol and cancer: Focus on in vivo evidence. Endocr Relat Cancer 21: R209-R225, 2014.

56. Howells LM, Berry DP, Elliott PJ, Jacobson EW, Hoffmann E, Hegarty B, Brown K, Steward WP and Gescher AJ: Phase I randomized, double-blind pilot study of micronized resveratrol (SRT501) in patients with hepatic metastases-safety, pharmacokinetics, and pharmacodynamics. Cancer Prev Res (Phila) 4: 1419-1425, 2011.

57. Patel KR, Brown VA, Jones DJ, Britton RG, Hemingway D, Miller AS, West KP, Booth TD, Perloff M, Crowell JA, et al: Clinical pharmacology of resveratrol and its metabolites in colorectal cancer patients. Cancer Res 70: 7392-7399, 2010.

58. Galiniak S, Aebisher D and Bartusik-Aebisher D: Health benefits of resveratrol administration. Acta Biochim Pol 66: 13-21, 2019.

(i) () () This work is licensed under a Creative Commons

Co Attribution-NonCommercial-NoDerivatives 4.0 International (CC BY-NC-ND 4.0) License. 